\title{
Force Sensorless Admittance Control with Neural Learning for Robots with Actuator Saturation
}

\author{
Guangzhu Peng, Chenguang Yang, Senior Member, IEEE, Wei He, Senior Member, IEEE, \\ and C. L. Philip Chen, Fellow, IEEE
}

\begin{abstract}
In this paper, we present a sensorless admittance control scheme for robotic manipulators to interact with unknown environments in the presence of actuator saturation. The external environment are defined as linear models with unknown dynamics. Using admittance control, the robotic manipulator is controlled to be compliant to external torque from the environment. The external torque acted on the end-effector is estimated by using a disturbance observer based on generalized momentum. The model uncertainties are solved by using radial basis neural networks. To guarantee the tracking performance and tackle the effect of actuator saturation, an adaptive neural network (NN) controller integrating an auxiliary system is designed to handle the actuator saturation is proposed. By employing Lyapunov stability theory, the stability of the closed-loop system is achieved. The experiments on Baxter robot are implemented to verify the the effectiveness of the proposed method.
\end{abstract}

Index Terms-adaptive neural control; observer; neural networks (NNs); admittance control

\section{INTRODUCTION}

$\mathbf{I}$ $\mathrm{N}$ the recent years, robots have been increasingly applied in a wide range of fields, such as elderly care, medical care and entertainment. In these cases, the robot will be faced with unknown and complex environment. Therefore, physical interaction with environment is an inevitable robot behaviour. The interactive behaviour of the robot may be the main objective of control design. Due to higher requirements for intelligence of robots, robots are expected to complete more difficult tasks on safety issues in social production life for human beings. Robots are required to learn and adapt to the environment to achieve compliant behaviours.

To make the robot better adapt to the unknown environment and achieve a compliant behavior, force sensing is essential and fundamental. Force sensing is a way to make a robot enable to detect objects near them. Traditional way to achieve force sensing is to use force sensors. Force sensors

This work was partially supported by Engineering and Physical Sciences Research Council (EPSRC) under Grant EP/S001913, National Natural Science Foundation of China under Grants 61751202 and 61572540 , Key Program for International S\&T Cooperation Projects of China under Gran 2016YFE0121200, Macau Science and Technology Development Fund (FDCT) under Grants 019/2015/A1, 079/2017/A2, and 024/2015/AMJ, Multiyear Research Grants of University of Macau. Corresponding author: C. Yang.

G. Peng is with the Department of Computer and Information Science, Faculty of Science and Technology, University of Macau, Macau 999078, China (Email: gz.peng@qq.com).

C. Yang is with Bristol Robotics Laboratory, University of the West of England, Bristol, BS16 1QY, UK (Email: cyang@iee.org).

W. He is with School of Automation and Electrical Engineering, University of Science and Technology Beijing, Beijing 100083, China (Email: hewei.ac@gmail.com).

C. L. Philip Chen is with the Faculty of Science and Technology, University of Macau, Macau 999078, China, with Department of Navigation, Dalian Maritime University, Dalian 116026, China, and also with the State Key Laboratory of Management and Control for Complex Systems, Institute of Automation, Chinese Academy of Sciences, Beijing 100080, China (e-mail: philip.chen@ieee.org). are always expensive, and will bring burden to the system integration. In addition, they could increase the complexity of task execution. Therefore, sensorless techniques have attracted more researchers. Early approaches of estimating the external force are introduced in [1] [2], and applied to the robotic manipulator [3]. Disturbance observer approaches based on control error for force estimation are often used in early robotic applications [4]. Recently, an alternative way is to use a force observer based on generalized momentum [5] [6]. The advantage of the generalized momentum based approaches is that joint acceleration is not needed. In [5], this method is further performed by a filtered model and a recursive leastsquares estimator. In [6], a Kalman filter is integrated in the generalized momentum approaches to estimate the contact forces/torques in Cartesian space.

Interaction control between robots and environments has been studied for long time and attracted much attention from a large number of researchers. Hybrid position/force control [7] is the most used method before impedance control proposed. However, when the environment is stiff, it may cause instability during the interaction. Impedance control aims to develop a relationship between the manipulator and the environment and is proved to have better robustness [8]. In the early literatures, researchers focused on dealing with uncertainties wth passive impedance models in robotic systems. Therefore, impedance control combining with adaptive control is often studied [9]. In [10], a desired impedance model is obtained with the consideration of environmental dynamics. Under impedance control, the manipulator could be compliant to the unknown environment [11]. Admittance control regarded as the inverse of impedance control is another scheme to achieve the compliant behavior [12]. Compared with impedance control methods, the concept of admittance control is that the system receives a force from the environment and exports a motion. Then, the compliant behavior of the manipulator will be achieved by trajectory adaptation to the environment [13]

Under admittance control, the tracking performance is important and essential after trajectory adaptation. It is well known that control strategies can be divided into two categories, namely model-based control and model-free control. Compared with model-free control, model-based control has a better control perfromance, but will depend heavily on the model accuracy. In practical systems, due to the existence of nonlinearities and uncertainties [14], perfect knowledge on the model cannot be assumed. Therefore, adaptive control methods integrating with intelligent architectures [15] [16], have been widely researched. Different from the traditional control methods, with powerful approximation ability, these adaptive methods do not require complete dynamics of robotic model [17]-[21]. In [22], in order to improve the dynamically substructured systems (DSS) testing performance, an adaptive 
NN-based controller is proposed and neural networks used to approximate uncertainties and nonlinearities on the DSS dynamics. In [23], a fuzzy logic system employed in backstepping control method is to approximate complicated functions. Evolutionary algorithms are also combined with fuzzy systems to achieve an optimal performance [24]. In [25], ant colony optimization and particle swarm optimization are integrated into fuzzy control systems to avoid the time-consuming task of manually designing the controllers.

In practical control systems, saturation is a common and unavoidable actuator nonlinearity and how to deal with actuator saturation is important. The saturation problem not only affects the control effect, but also may lead to the instability [26]. Therefore, effort of investigation has been considered on this topic. Based on adaptive control theory, several derived adaptive schemes to solve the saturation problems have been studied to handle actuator saturation [27]. In recent years, neural learning adaptive schemes have received much attention [28]. In [29], based on the state observer, neural networks are employed into control design to deal with the effects of the unknown disturbances and the saturation nonlinearity. In [30], a well defined smooth function and a Nussbaum function are integrated into adaptive control design. The saturation effect will lead to nonlinear terms, which is compensated by the Nussbaum function. In [31], an adaptive neural impedance control is designed for a $n$-link robotic manipulator with input saturation. An auxiliary system is introduced in control design to deal with the saturation effect. This paper is a continuation of our previous work [32], and the contributions are summarized as follows:

(i) Admittance control method has been employed to achieve a compliant behaviour with the consideration of environmental dynamics in robot-environment systems.

(ii) An RBFNN-based controller integrating an auxiliary system is designed in the presence of actuator saturation and uncertainties in robotic system.

(iii) The external torque in the admittance model in joint space is estimated by a torque observer replacing force sensors to reduce the system burden.

The rest of the paper is organized as follow. In Section II, problem statement and preliminaries are presented. In Section III, the admittance control design with neural networks in the presence of input saturation is discussed. In Section IV, experimental results are presented. The appendix is the final section of the paper and follows the conclusion.

\section{PROBLEM STATEMENT AND PRELIMINARIES}

\section{A. Problem Formulation}

Generally, most of the environmental dynamics can be expressed as [33]

$$
\begin{aligned}
& M_{E} \ddot{q}+C_{E} \dot{q}+G_{E} q=-\tau_{e} \\
& C_{E} \dot{q}+G_{E} q=-\tau_{e}
\end{aligned}
$$

where $M_{E}, C_{E}$ and $G_{E}$ denote mass, damping and stiffness respectively.

As shown in Fig. 1, considering a robot arm interacting with an unknown environment, a control scheme is designed to make the robot arm have a compliant behaviour, and will satisfy the following requirements: i) based on the admittance method, the desired trajectory will be modified when an external force is acting on the robot arm; ii) the external torque applied at end-effector is estimated by the observer; iii) the adaptive neural controller can guarantee the tracking performance.

\section{B. System Dynamics}

The robot kinematics is given as follows

$$
x(t)=\phi(q)
$$

where $x(t)$ is the vector of position and orientations and $q$ is the vector of joint angles. Then, the inverse kinematics are

$$
q(t)=\phi^{-1}(x)
$$

Take the derivative of (2) with respect to time, we have

$$
\dot{x}(t)=J(q) \dot{q}
$$

where $J(q)$ is the Jacobian matrix. Further differentiating (4), we have

$$
\ddot{x}(t)=\dot{J}(q) \dot{q}+J(q) \ddot{q}
$$

The relationship between joint force and wrench is

$$
\tau_{\text {ext }}=J^{\mathrm{T}}(q) f
$$

Then, we give the $n$-link robot manipulator dynamics in joint space

$$
D_{q}(q) \ddot{q}+C_{q}(q, \dot{q}) \dot{q}+G_{q}(q)+\tau_{\text {fric }}=\tau+\tau_{\text {ext }}
$$

where $q \in \mathbb{R}^{n}, \dot{q} \in \mathbb{R}^{n}$ and $\ddot{q} \in \mathbb{R}^{n}$ are the vectors of joint angle, velocity and accelerations respectively. $D_{q}(q) \in \mathbb{R}^{n \times n}$ denotes the inertia matrix; $C_{q}(q, \dot{q}) \dot{q}$ denotes the Coriolis and centripetal torque; $G_{q}(q)$ is the gravity. $\tau$ is the robot motor torque; $\tau_{\text {fric }}$ is the friction torque and $\tau_{\text {ext }}$ is the external torque.

a) Property 1 [34]: Matrix $D_{q}(q)$ is symmetric and positive definite.

b) Property 2 [34]: Matrix $\dot{D}_{q}(q)-2 C_{q}(q, \dot{q})$ is a skewsymmetric matrix.

An admittance model describes the relationship between the external force and position of robot arm [35]

$$
M_{d}\left(\ddot{x}_{r}-\ddot{x}_{d}\right)+C_{d}\left(\dot{x}_{r}-\dot{x}_{d}\right)+K_{d}\left(x_{r}-x_{d}\right)=-f
$$

where $x_{d} \in \mathbb{R}^{n}$ is the desired trajectory, and $x_{r} \in \mathbb{R}^{n}$ is the virtual desired trajectory arised from the external force $f$.

Substituting (2)-(5) into (8), the left side of (8) is

$$
\begin{aligned}
& M_{d}\left(\ddot{x}_{r}-\ddot{x}_{d}\right)+C_{d}\left(\dot{x}_{r}-\dot{x}_{d}\right)+K_{d}\left(x_{r}-x_{d}\right) \\
& =M_{d} J(q)\left(\ddot{q}_{r}-\ddot{q}_{d}\right)+\left(C_{d} J(q)+M_{d} \dot{J}(q)\right)\left(\dot{q}_{r}-\dot{q}_{d}\right) \\
& \quad+K_{d}\left(\phi\left(q_{r}\right)-\phi\left(q_{d}\right)\right)
\end{aligned}
$$

Then the admittance model in joint space can be defined as

$$
\begin{aligned}
M_{d} J(q)\left(\ddot{q}_{r}-\ddot{q}_{d}\right) & +\left(C_{d} J(q)+M_{d} \dot{J}(q)\right)\left(\dot{q}_{r}-\dot{q}_{d}\right) \\
& +K_{d}\left(\phi\left(q_{r}\right)-\phi\left(q_{d}\right)\right)=-J^{-T} \tau_{\text {ext }}
\end{aligned}
$$

where $q_{d} \in \mathbb{R}^{n}$ and $q_{r} \in \mathbb{R}^{n}$ are the desired trajectory and virtual desired trajectory in the joint space, respectively. The $M_{d}, C_{d}$ and $K_{d}$ are gain matrices denoting the mass, damping and stiffness matrix specified by the designer.

Assumption 1: Both $q_{d}$ and $q_{r}$ are bounded and differentiable: $\left\|q_{d}\right\|,\left\|q_{r}\right\| \leq c_{1},\left\|\dot{q}_{d}\right\|,\left\|\dot{q}_{r}\right\| \leq c_{2},\left\|\ddot{q}_{d}\right\|,\left\|\ddot{q}_{r}\right\| \leq c_{3}$ and $c_{1}, c_{2}$ and $c_{3}$ are positive constants. 


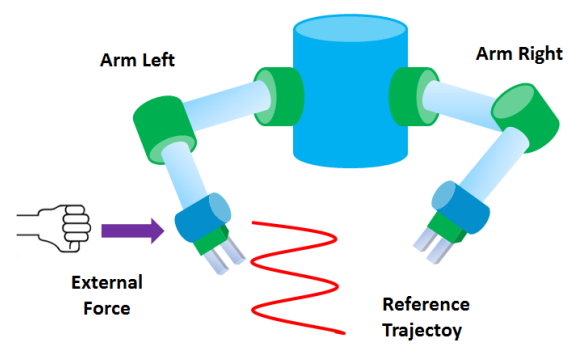

Fig. 1. The overview of robot arm interacting with environment

Remark 1: In some specific situations, other admittance models such as damping-stiffness model and stiffness model are given

$$
\begin{aligned}
C_{d}\left(\dot{q}_{r}-\dot{q}_{d}\right)+K_{d}\left(q_{r}-q_{d}\right) & =-\tau_{\text {ext }} \\
K_{d}\left(q_{r}-q_{d}\right) & =-\tau_{\text {ext }}
\end{aligned}
$$

We can find that if there is no external collision and the desired manipulator's motion is free, we have $q_{r}=q_{d}, \tau_{\text {ext }}=$ 0 . On the contrary, when external collision exists, the robot arm will follow the new trajectory which can be seen as the adaptation to the external torque and the target admittance model defined in (10) describes this relationship.

\section{Actuator Saturation}

Saturation is a static nonlinear function used to describe the insensitivity of large signals which exceed the input limit of the actuator, as shown in Fig. 2.

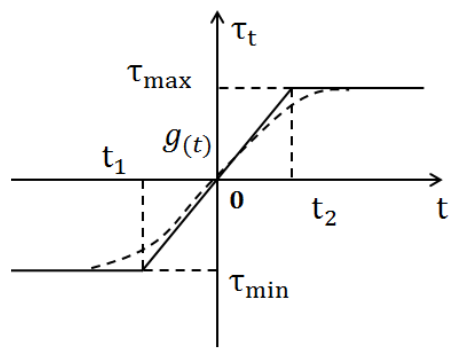

Fig. 2. The saturation nonlinearity

In general, the saturation can be described as

$$
S_{a t}(\tau)= \begin{cases}\tau_{\max } & u \geq \tau_{\max } \\ g(t) & \tau_{\min }<u<\tau_{\max } \\ \tau_{\min } & u \leq \tau_{\min }\end{cases}
$$

where $u$ is the input signal, $g(t)$ is a smooth function; $S_{a t}(\tau)$ is the output of the saturation nonlinearity; $\tau_{\max }$ and $\tau_{\min }$ denote the maximum and minimum value of saturation nonlinearity, respectively.

\section{NN Approximation}

With the approximation capability of the RBFNN [36], a continuous smooth function $h(Z): \mathbb{R}^{q} \rightarrow \mathbb{R}$ is defined, and RBFNN is used to approximate it

$$
h_{n n}\left(Z_{i n}\right)=W^{\mathrm{T}} S\left(Z_{i n}\right)
$$

where $Z_{\text {in }} \in \Omega \subset R^{q}$ denotes the input of RBFNN; $W=\left[w_{1}, w_{2}, \ldots, w_{m}\right] \in R^{m}$, denotes the NN weight and $m>0$ is NN node number in the hidden layer; $S\left(Z_{i n}\right)=$ $\left[S_{1}\left(Z_{i n}\right), S_{2}\left(Z_{i n}\right), \ldots, S_{m}\left(Z_{i n}\right)\right]^{\mathrm{T}}$ and $S_{i}\left(Z_{i n}\right)$ denotes an activation function which is often chosen as Gaussian function

$$
S_{i}\left(Z_{\text {in }}\right)=\exp \left[\frac{-\left(Z_{\text {in }}-u_{\mathrm{i}}^{\mathrm{T}}\right)\left(Z_{\text {in }}-u_{\mathrm{i}}\right)}{\eta_{\mathrm{i}}^{2}}\right], \mathrm{i}=1, \ldots, \mathrm{m}
$$

where $u_{i}=\left[u_{i 1}, u_{i 2}, \ldots, u_{i q}\right]^{\mathrm{T}} \in R^{q}$ is the center of receptive field and $\eta_{i}$ is the variance. From the definition of activation function, we can obtain that the $S\left(Z_{i n}\right)$ is bounded, which can be described as

$$
\left\|S\left(Z_{\text {in }}\right)\right\| \leq \varrho
$$

where $\varrho$ is a positive constant.

With a sufficiently large node $m$, any smooth continuous function can be approximated to any degree

$$
h_{n n}\left(Z_{i n}\right)=W^{* \mathrm{~T}} S\left(Z_{i n}\right)+\varepsilon
$$

where $W^{*}$ is the ideal weight over a compact set $\Omega_{Z_{\text {in }}} \subset R^{q}$; the approximation error of RBFNN satisfies $\|\varepsilon\| \leq \omega$, where $\omega$ is a small unknown constant. Over a compact set $Z_{\text {in }} \in$ $\Omega_{Z_{\text {in }}} \subset R^{q}$, the ideal weight vector can be

$$
\left.W^{*}=\arg \min _{Z_{i n} \subset R^{m}}\left\{\sup \mid h_{n n}\left(Z_{i n}\right)-W^{\mathrm{T}} S\left(Z_{i n}\right)\right) \mid\right\}
$$

\section{CONTROL STSTEM DESIGN AND STABILITY ANALYSIS}

In this section, an admittance control scheme is developed, as shown in Fig. 3. The collision from the environment is viewed as the external torque exerted at the end-effector, which is estimated by observer approach and also seen as the disturbance of the system.

\section{A. Observer based on the generalized momentum}

Traditional force estimation methods rely on the model of the manipulator involve joint acceleration $\ddot{q}$ and the inverse of mass matrix $D_{q}(q)$ [37], which will bring the amplification of measurement noise to the system and increase the amount of calculation of the system. To solve this problem, a disturbance observer based on the generalized momentum is developed [5]. This approach can be used to estimate the external torque without involving joint acceleration $\ddot{q}$ and computation of the inverse of the matrix $D_{q}(q)$.

The generalized momentum of robot joint can be described as

$$
p=D_{q}(q) \dot{q}
$$

and its derivative is

$$
\dot{p}=\dot{D}_{q} \dot{q}+D_{q} \ddot{q}
$$

Considering the robot dynamics (7), (19) can be written as $\dot{p}=\dot{D}_{q}(q, \dot{q}) \dot{q}+\tau-C_{q}(q, \dot{q}) \dot{q}-G_{q}(q)+\tau_{\text {ext }}-\tau_{\text {fric }}$

Using the property 2 with a symmetry $D_{q}(q),(20)$ can be

$$
\begin{aligned}
\dot{p} & =\dot{D}_{q}(q, \dot{q}) \dot{q}+\tau-C_{q}(q, \dot{q}) \dot{q}-G_{q}(q)+\tau_{\text {ext }}-\tau_{f r i c} \\
& =\tau+C_{q}^{\mathrm{T}}(q, \dot{q}) \dot{q}-G_{q}(q)+\tau_{\text {ext }}-\tau_{\text {fric }}
\end{aligned}
$$




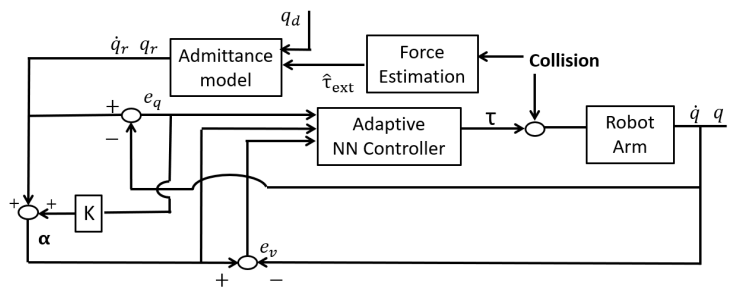

Fig. 3. The overview of the control scheme

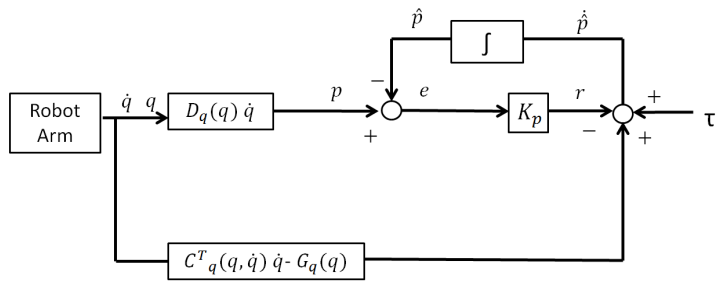

Fig. 4. The overview of the observer based on the generalized momentum

It can be seen that, in (21), the derivative of $p$ depends on the external torque $\tau_{\text {ext }}$ and no acceleration term of robot joints.

Now, an observer is defined for generalized momentum $p$, as shown in Fig. 4. The error of the $p$ is defined as

$$
e_{p}=p-\hat{p}
$$

Then, we have

$$
\dot{\hat{p}}=\tau+C_{q}^{\mathrm{T}}(q, \dot{q}) \dot{q}-G_{q}(q)-\tau_{f r i c}+K_{p} e_{p}
$$

where $K_{p}$ is the positive gain matrix.

In this paper, we use the model of Baxter robot in [38]. As we can see, combine (21) with (23) and define $r=K_{p} e_{p}$, we can obtain

$$
\begin{aligned}
\dot{r} & =K_{p}(\dot{p}-\dot{\hat{p}}) \\
& =-K_{p}\left(-\tau_{\text {ext }}+r\right)
\end{aligned}
$$

Remark 2: In robot-environment interaction applications, control of contacted force is essential and important. The force observer is a feasible way to estimate external forces using the dynamics of the system. However, due to the uncertainties in system dynamics, these model-based observers can not provide accurate force estimates when the dynamics of the model are not accurate. One straightforward solution is to use adaptive methods to estimate unknown parameters. The adaptive estimation technique has been investigated in previous literatures, e.g., in [5], an adaptive parameters estimation method was designed to estimate the unknown robot dynamics and a recursive least squares estimation algorithm is proposed for external force without the joint acceleration $\ddot{q}$. With the powerful approximation ability of neural networks, many works have been investigated to use neural-networkbased force/torque observers for estimating the contact force and achieve a good performance [39] [40]. These NN-based observers have the advantage of knowing little information about the robots dynamics and avoid the restriction on traditional model-based observer approaches. In this regard, modelfree observer integrating in NN-based adaptive control scheme will be included in our future work.

\section{B. Controller}

In this section, we develop a controller to track the desired trajectory with input constraints. After that, stability analysis will be presented. First, in joint space, we define some related error signals as follows

$$
\begin{aligned}
& e_{q}=q_{r}-q \\
& \alpha=\dot{q}_{r}+K e_{q} \\
& e_{v}=\dot{e}_{q}+K e_{q}
\end{aligned}
$$

where $K$ is positive gain matrix. Taking the external disturbance into consideration, we define the control torque

$$
\begin{aligned}
\tau=-e_{q}+\hat{D}_{q} \dot{\alpha}+\hat{C}_{q} \alpha+\hat{G}_{q}-\hat{\tau}_{e x t} & \\
& +K_{v}\left(e_{v}+\xi\right)+K_{s} \operatorname{sgn}\left(e_{v}\right)
\end{aligned}
$$

where $K_{v}$ and $K_{s}$ are gain matrices; $\hat{D}_{q}, \hat{C}_{q}$ and $\hat{G}_{q}$ are the approximations of RBFNN; $\xi$ is the state variable which will be defined latter.

Considering the dynamics of a robot system with actuator saturation

$$
D_{q}(q) \ddot{q}+C_{q}(q, \dot{q}) \dot{q}+G_{q}(q)=S_{a t}(\tau)+\tau_{\text {ext }}
$$

where $S_{a t}(\bullet)$ is the function of actuator saturation defined in (12). Substituting (26) into (27), we have

$$
\begin{aligned}
-D_{q} \dot{e}_{v}= & \left(\hat{D}_{q}-D_{q}\right) \dot{\alpha}+\left(\hat{C}_{q}-C_{q}\right) \alpha+\left(\hat{G}_{q}-G_{q}\right) \\
& -e_{q}+C_{q} e_{v}+\Delta \tau+\Delta \tau_{e} \\
& +K_{v}\left(e_{v}+\xi\right)+K_{s} \operatorname{sgn}\left(e_{v}\right)
\end{aligned}
$$

where

$$
\begin{aligned}
& \Delta \tau=S_{a t}(\tau)-\tau \\
& \Delta \tau_{e}=\tau_{e x t}-\hat{\tau}_{e x t}
\end{aligned}
$$

The auxiliary system is defined as

$\dot{\xi}= \begin{cases}-K_{\xi} \xi-\frac{\left|e_{v}^{\mathrm{T}} \Delta \tau\right|+0.5 \Delta^{\mathrm{T}} \tau \Delta \tau}{\|\xi\|^{2}}+\Delta \tau & \text { if }\|\xi\|>\mu \\ 0 & \text { if }\|\xi\| \leq \mu\end{cases}$

where $K_{\xi}$ is a positive gain matrix and $\mu$ is defined as a small constant.

Employing the approximation of neural networks, we can obtain

$$
\begin{array}{r}
\hat{D}_{q}(q)=\hat{W}_{D}^{\mathrm{T}} S_{D}(q) \\
\hat{C}_{q}(q, \dot{q})=\hat{W}_{C}^{\mathrm{T}} S_{C}(q, \dot{q}) \\
\hat{G}_{q}(q)=\hat{W}_{G}^{\mathrm{T}} S_{G}(q)
\end{array}
$$

The updating laws of RBFNN are

$$
\begin{aligned}
& \dot{\hat{W}}_{D}=\Theta_{D}\left(S_{D} \dot{\alpha} e_{v}-\delta_{D} \tilde{W}_{D}\right) \\
& \dot{\hat{W}}_{C}=\Theta_{C}\left(S_{C} \alpha e_{v}-\delta_{C} \tilde{W}_{C}\right) \\
& \dot{\hat{W}}_{G}=\Theta_{G}\left(S_{G} e_{v}-\delta_{G} \tilde{W}_{G}\right)
\end{aligned}
$$

where $\Theta_{D}, \Theta_{C}$ and $\Theta_{G}$ are positive matrices, $\delta$ is a small gain matrix for disturbance [41]. Then, the dynamics (28) can be derived into

$$
\begin{aligned}
-D_{q} \dot{e}_{v}= & -e_{q}+C_{q} e_{v}+\Delta \tau+\Delta \tau_{e}+K_{v}\left(e_{v}+\xi\right) \\
& +K_{s} \operatorname{sgn}\left(e_{v}\right)+\left(\hat{W}_{D}-W_{D}\right) S_{D} \dot{\alpha}+\left(\hat{W}_{C}-W_{C}\right) S_{C} \alpha \\
& +\left(\hat{W}_{G}-W_{G}\right) S_{G}
\end{aligned}
$$




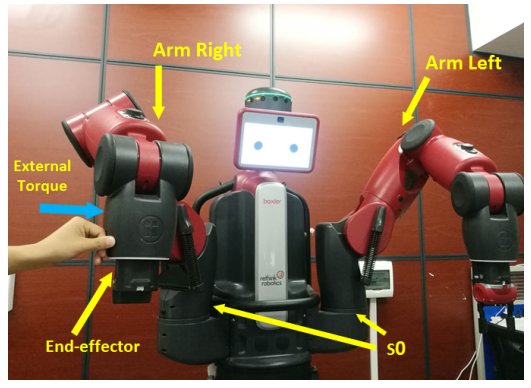

Fig. 5. The illustration of the experiment.

then

$$
\begin{aligned}
-D_{q} \dot{e}_{v}= & -e_{q}+C_{q} e_{v}+\Delta \tau+\Delta \tau_{e}+K_{v}\left(e_{v}+\xi\right) \\
& +K_{s} \operatorname{sgn}\left(e_{v}\right)+\tilde{W}_{D}^{\mathrm{T}} S_{D} \dot{\alpha}+\tilde{W}_{C}^{\mathrm{T}} S_{C} \alpha+\tilde{W}_{G}^{\mathrm{T}} S_{G}
\end{aligned}
$$

where $\tilde{W}_{D}=\hat{W}_{D}-W_{D}, \tilde{W}_{C}=\hat{W}_{C}-W_{C}$ and $\tilde{W}_{G}=$ $\hat{W}_{G}-W_{G}$

Theorem 1: Consider the definition of $V$, we can obtain $e_{q}, e_{v}, \xi,\left\|\tilde{W}_{D}\right\|,\left\|\tilde{W}_{C}\right\|$ and $\left\|\tilde{W}_{G}\right\|$ are uniformly ultimately bounded. Since $\|W\|$ is bounded, $\|\hat{W}\|=\|W+\tilde{W}\|$ is bounded. With the given bounded $q_{d}, \dot{q}_{d}, q_{r}$ and $\dot{q}_{r}$, according to the definition of error signals in (25), we can obtain $q=q_{r}-e_{q}$ is bounded; $\alpha=\dot{q}_{r}+K e_{q}$ is bounded.

Remark 3: As well known, Lyapunov direct method is a very important controller design and stability analysis tool in nonlinear systems. By constructing a Lyapunov function and analyzing its derivative with respect to time, the stability at the equilibrium point can be obtained without seeking the system solution. Given a nonlinear dynamic system

$$
\dot{x}=f(x, t), x(0)=x_{0}
$$

where $x \in \mathbb{R}^{n}$. Its equilibrium point is the origin. $N$ is the neighborhood of the origin, where $N=x:\|x\| \leq \epsilon, \epsilon>0$. Then, We can analyze the convergence of system states by constructing a scalar Lyapunov function. However, lyapunov method also has its limitations in some situations. In general, the lyapunov stability analysis method focuses on the final convergence results of the system state, that is, whether the state converges or not. It rarely pays attention to convergence process of system states. For example, in a practical control system, we can use lyapunov direct method to analyze whether the control errors of the system converge, but some transient target, such as the overshoot and rise time, are difficult to be achieved. Even in some practical cases, the lyapunov method can lead to infeasible controller design and failing to achieve a desired performance [42]. Another conservative point of lyapunov method is that only quadratic Lyapunov functions are considered in most cases. some related strategies to reduce the conservatism are studied in [17], [43]-[45].

\section{EXPERIMENT RESULTS}

To illustrate effectiveness of our developed method, we utilize the Baxter robot to perform the experiments. The Baxter robot has two arms and each of the Baxter robot has 7 Degrees of Freedom (DOF): shoulder joints $s_{0}, s_{1}$, elbow joints: $e_{0}$, $e_{1}$ and wrist joints: $w_{0}, w_{1}$ and $w_{2}$. The model of the Baxter is introduced in [38].
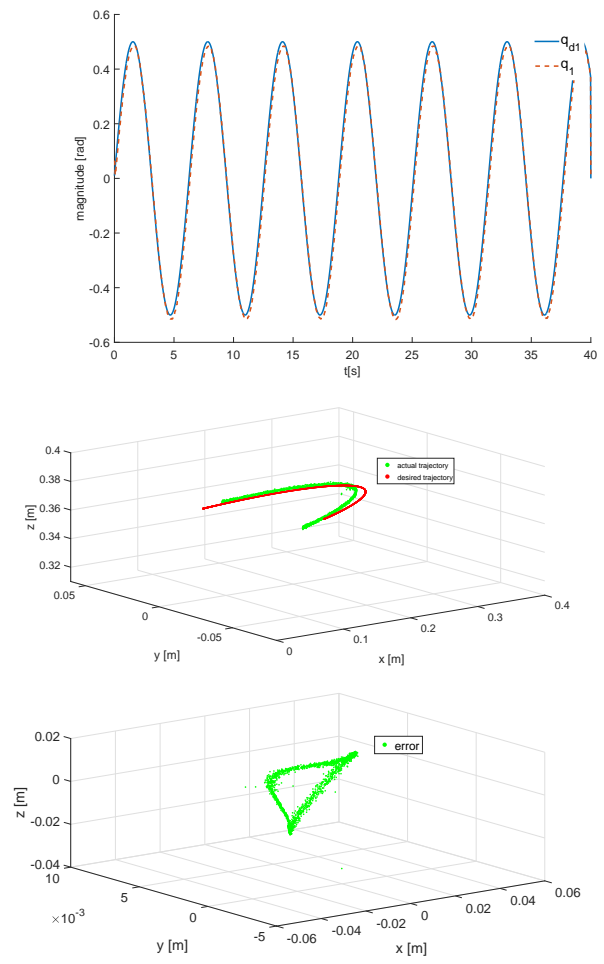

Fig. 6. The tracking performance of NN controller.

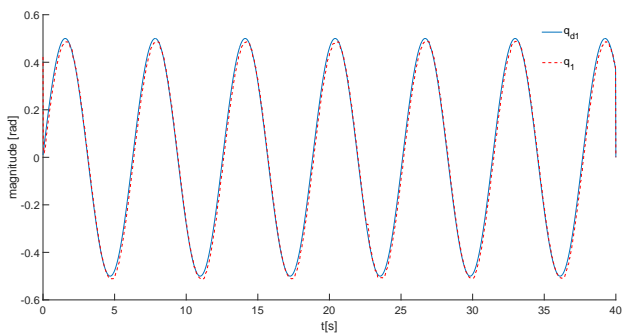

Fig. 7. The tracking performance with input saturation.

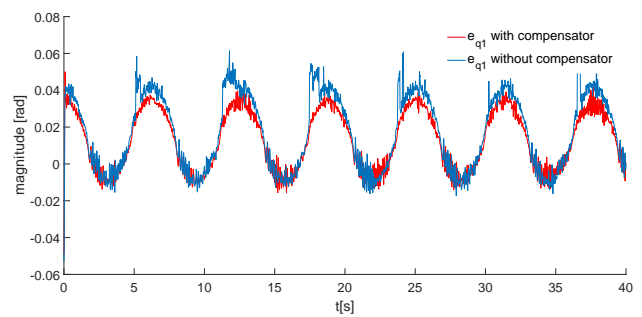

Fig. 8. The tracking error of joint $s_{0}$.

In this experiment, at the beginning, when there is no interaction with the external environment, the robot manipulator will follow the desired trajectory. After a period of time, an external torque will be applied at the end-effector when the robot begins to interact with the environment. Under the influence of external torque, the reference trajectory $q_{d}$ of the robot will be modified to adapt to the environment and a modified trajectory $q_{r}$ will be generated. The modified trajectory $q_{r}$ is viewed as the adaptation behaviour of the robot manipulator to the environment. The description of 


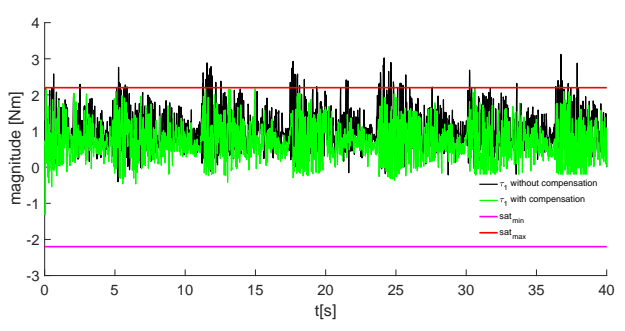

Fig. 9. The control input of joint $s_{0}$ with/without saturation compensator.

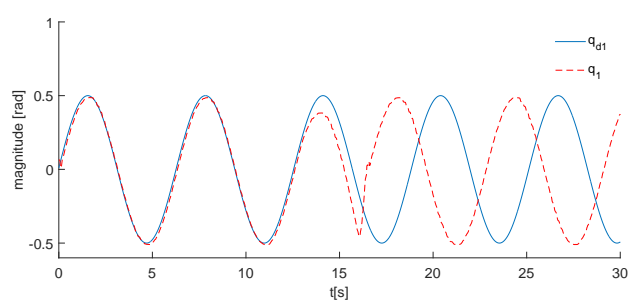

Fig. 10. The trajectory adaptation for external torque.

the experiment is depicted in Fig. 5. In the course of the experiment, we test the shoulder joint $s_{0}$.

\section{A. Test of NN Controller}

First, we test the performance of the adaptive NN controller. The desired trajectory of the manipulator: $q_{d}=[0.5 \sin (\mathrm{t})$; $1 ; 1.19 ; 1.94 ;-0.67 ; 1.03 ;-0.5]$. The initial position of the manipulator: $q_{0}=[0.06 ;-1 ; 1.19 ; 1.94 ;-0.67 ; 1.03 ;-0.5]$ and initial velocity is set: $\dot{q}_{0}=[0 ; 0 ; 0 ; 0 ; 0 ; 0 ; 0]$. We employ $7 \mathrm{NN}$ nodes for each input dimension to approximate uncertainty and the initial configuration of $\mathrm{NN}$ are $\hat{W}_{D_{q}}(0)=0$, $\hat{W}_{\boldsymbol{C}_{\boldsymbol{q}}}(\mathbf{0})=\mathbf{0}$ and $\hat{W}_{\boldsymbol{G}_{\boldsymbol{q}}}(\mathbf{0})=\mathbf{0}$. The gains of $\mathrm{NN}$ law are $\Theta_{D}=\operatorname{diag}[0.01,0.01,0.01,0.01,0.01,0.01,0.01], \delta_{D}=$ $\operatorname{diag}[15,15,15,15,15,15,15]$. The control gains are set $K=$ $\operatorname{diag}[10,10,10,10,10,10,10], K_{v}=\operatorname{diag}[5,6,5,4,1,1,1]$ and $K_{s}=\operatorname{diag}[2,2,2,2,2,2,2]$.

The tracking performance is depicted in Fig. 6. As shown in Fig. 6, the actual trajectories of the end-effector and the joint $s_{0}$ can follow the desired trajectories effectively, and the average tracking errors of the end-effector with respect to $x, y, z$ are around $(-0.03 m, 0.04 m),(0.004 m, 0.008 m),(-0.003 m, 0.007 m)$, respectively, where $(\bullet)$ denotes the range of values. The overall results are satisfactory, which implies the effectiveness of the adaptive $\mathrm{NN}$ controller.

\section{B. Test of Saturation Compensator}

This group of experiments aim at testing the effectiveness of the saturation compensator on joint $s_{0}$. The parameters are selected as $K_{\xi}=\operatorname{diag}\{25 ; 0 ; 0 ; 0 ; 0 ; 0 ; 0\}$, sat $_{\text {max }}=\operatorname{diag}\{2.2 ; 5 ; 5 ; 5 ; 5 ; 5 ; 5\}$ and sat $_{\text {min }}=$ $\operatorname{diag}\{-2.2 ;-5 ;-5 ;-5 ;-5 ;-5 ;-5\}$. where $K_{\xi}$ is the gain matrix in auxiliary system for saturation compensation and $s a t_{\max }, s a t_{\min }$ are virtual saturation limits. When the amplitude of the control input is larger than the virtual saturation limits, it will be set equal to the amplitude of virtual saturation limits.

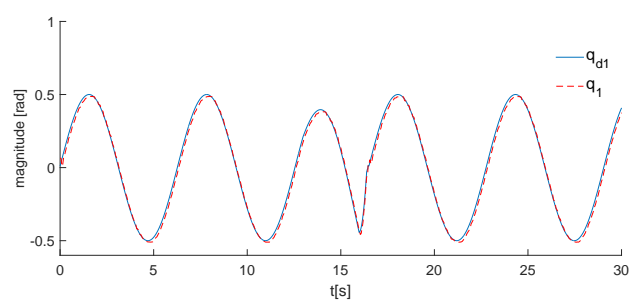

Fig. 11. The tracking performance of joint $s_{0}$.

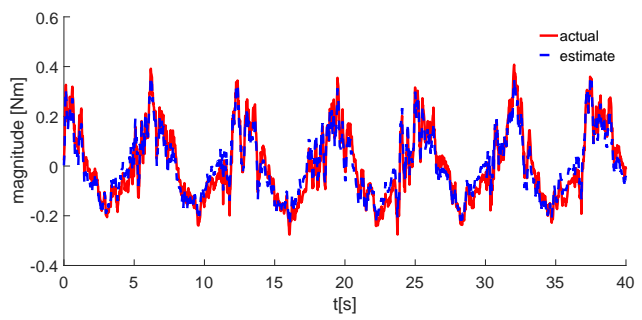

Fig. 12. The estimation performance of observer approach.

The experimental results are illustrated in Figs. 7-9. As shown in Fig. 9, when there is no saturation compensation, the amplitude of control input is larger than the upper bound at some points. With the compensator, we can find that, the points of input torque which are larger than the upper bound are compensated, so the input of the torque can be limited within the upper and lower bounds. In Fig. 7, the tracking performance with input compensation is presented and the tracking errors are also compared with and without the input compensation in Fig. 8. As shown in Fig. 8, without compensation, the tracking errors are larger because the actuator cannot provide enough energy to guarantee the tracking performance where the points of control input reach out the saturation limits. The comparative figures show that, with input compensation, the controller can ensure good position tracking performance, which demonstrate that our control strategy is effective.

\section{Test of Admittance Control}

This group of experiments aims at testing the effectiveness of torque estimation and trajectory adaptation for the environment. The external torque is defined as $\tau_{\text {ext }}=0.3 \dot{q}+0.2 q$. The mass, damping and stiffness matrices in admittance model are set $M_{d}=$ $\operatorname{diag}[5,5,5,5,5,5,5], C_{d}=\operatorname{diag}[10,10,10,10,10,10,10]$ and $K_{d}=\operatorname{diag}[20,20,20,20,20,20,20]$. The gain of the observer is set $K_{p}=\operatorname{diag}[3.2,3.2,3.2,3.2,3.2,3.2,3.2]$.

The experiment results are shown in Figs. 10-12. The acting time of the external force is from $13 \mathrm{~s}$ to $15 \mathrm{~s}$. As shown in Fig. 10, when the manipulator is interacting with the environment, the desired trajectory will be modified to adapt to the environment. By using a filter to make the result clearer, the estimation performance is demonstrated in Fig. 12, which shows a satisfactory results. The tracking performance for the modified trajectory is illustrated in Fig. 11. The overall results demonstrate that the proposed control scheme is effective. 


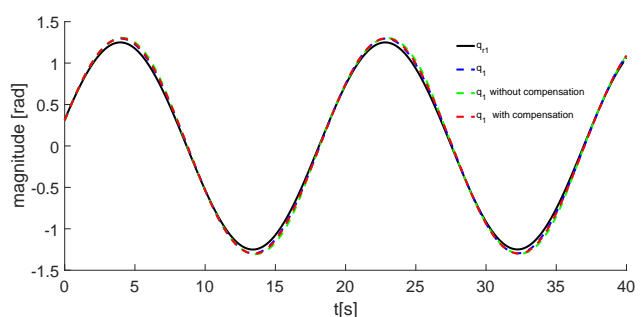

Fig. 13. Tracking performance of Joint 1 .

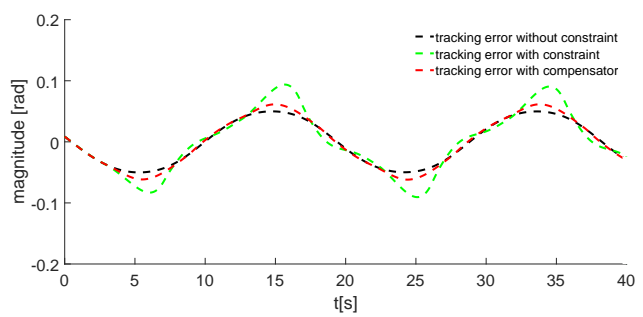

Fig. 14. Tracking errors of Joint 1.

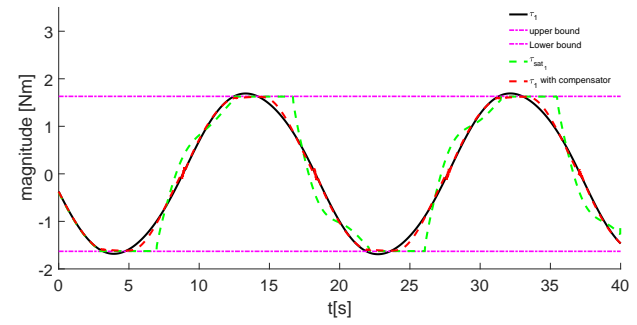

Fig. 15. Control torques of Joint 1.

\section{Discussion}

Comparative simulation studies are conducted to further verify the proposed method. The first group of comparative simulation is carried out to illustrate the influence of input saturation restriction on control performance in admittance control scheme. In [46], an adaptive admittance control method for human-robot interaction is developed, and the inner control loop is to guarantee the actual trajectory $x$ can track the desired trajectory $x_{m}$ generated from an admittance model without input constraints. The comparative simulations are taking the input saturation into consideration, and results are depicted in Figs. 13-15, where Figs. 13-14 are the tracking performance and Fig. 15 is the control inputs of joint 1 under different conditions. The desired trajectory of joint 1 is $q_{d}=1.25 \sin (0.25 \mathrm{t})$, and initial condition of the joint is $q_{0}=0.3, \dot{q}_{0}=0$. The upper saturation bound is set $1.65 \mathrm{Nm}$ and the lower is $-1.65 \mathrm{Nm}$. Three situations are considered in the simulation: no saturation constraint (black line), saturation without compensation (green dashed-dotted line), saturation with compensation (red dashed-dotted line). From Figs 13-14, we can find that the tracking performance of the controller with saturation compensation is better than the controller without saturation compensation which degrades in the case of saturation constraints. Furthermore, by employing the compensation technique in the controller, the control

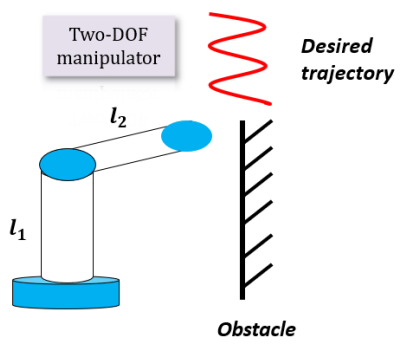

Fig. 16. Simulation model modified from [31].

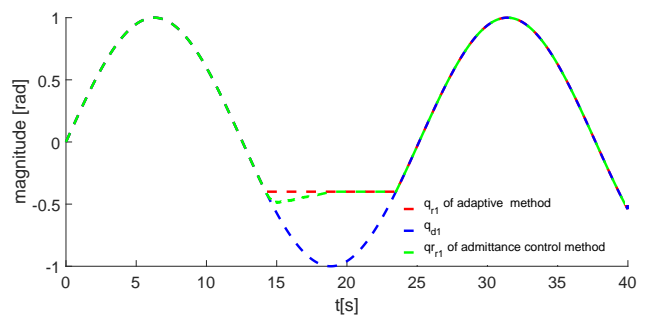

Fig. 17. Trajectory of each method.

performance can be improved without exceeding the upper and lower bounds of the input of the actuator, as shown in Fig. 15. It can be referred from that, in practical admittance control scheme, when the actuator is saturated, it is difficult to ensure that the robot can track the desired trajectory obtained from the admittance model, which results in that the ultimate control goal in [46] may not be achieved.

To further validate the effectiveness of admittance control method in interactive control system, another comparative studies are designed based on the admittance method and the adaptive control method in [31]. In [31], a desired impedance model and the external force $\tau_{e}$ are assumed to be completely known, which are ideal conditions in practice. Therefore, we conduct the simulation under the same environment and remove these ideal conditions. The desired trajectory and the initial configuration of joint 1 is set $q_{d}=1.25 \sin (0.25 t)$, and initial joint condition is $q_{0}=0, \dot{q}_{0}=0$. As depicted in Fig. 16, the manipulator is designed to follow the desired trajectory $q_{d}$. After a period of time, the manipulator will have a collision with the obstacle. Simulation results are presented in Figs. 17-19. As shown in Fig. 17, after a collision, by using admittance control method (trajectory of green dasheddotted line), the desired trajectory (blue dashed-dotted line) set by the designer will be modified to reduce the contact torque and have a compliant behaviour. On the contrary, the desired trajectory will not be modified by using the adaptive control method (trajectory of red dashed-dotted line), and the manipulator is tracking the desired trajectory all the time. Thus, the interaction torque (red solid line) is increasing in the course of collision by adaptive control method without ideal assumptions, and the contact torque (green dashed-dotted line) by using admittance control method is smaller, as shown in Fig. 19. The tracking performance of both methods is depicted in Fig. 18. From these comparative results, we can find that admittance control method combining with observer approach is more applicable and can make the robotic manipulator have 


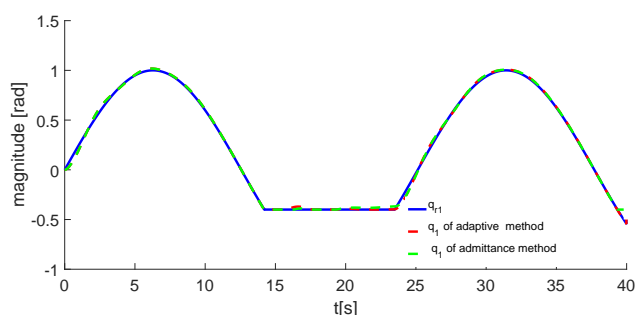

Fig. 18. Tracking performance of each method.

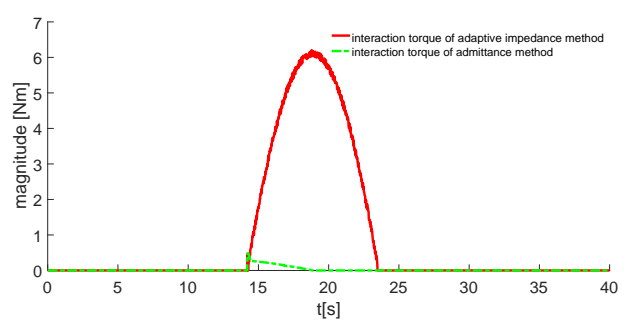

Fig. 19. The interaction torque of each method.

a compliant behaviour to external torque/force.

In fact, the proposed sensorless admittance control scheme also has some weak points. As pointed out, in practical system, some noise in the observer approach will have a inverse influence on the estimation accuracy, and an expected modified desired-trajectory obtained from the admittance model in (11) could not be guaranteed. From the point of estimation accura$\mathrm{cy}$, in [46], a force sensor is used to measure the contact force could be closer to real value of torque/force. Furthermore, due to the uncertainties of the model dynamics, the generalized momentum based observer may meet some limitations in certain practical applications and this will be included in our future work to expand the scope of applications of our proposed control scheme. In addition, input saturation does not cover all the non-linear phenomena in actual mechanical systems, and other constraints (e.g. dead-zone and time-delay) should be further considered.

\section{CONCLUSION}

This paper presents a sensorless control scheme integrating RBFNN, torque estimation and admittance control for Baxter robot to interact with the unknown environment with input constraint. The adaptive neural controller can guarantee the tracking performance and the tracking errors of the system within a small neighborhood of zero. The external torque from the environment applied at end effector is estimated and admittance control method is employed for trajectory adaptation to achieve a compliant behaviour. Finally, the experiment results on Baxter robot demonstrate the effectiveness of the proposed method.

In the future work, many other non-linear constraint problems (e.g. dead-zone, time-delay and hysteresis) will be considered in our proposed system. In these situations, the performance and stability of the system might not be guaranteed. Furthermore, analysis and studies on dealing with more complex environmental models will enrich the diversity of the admittance control system. In addition, the proposed NN-based adaptive control scheme can be combined with free-model observer using intelligent tools, such as radial basis function neural network (RBFNN), together to make the control scheme more applicable.

\section{APPENDIX}

Considering the Lyapunov candidate

$$
\begin{aligned}
V & =\frac{1}{2} e_{q}^{\mathrm{T}} e_{q}+\frac{1}{2} e_{v}^{\mathrm{T}} D_{q} e_{v}+\frac{1}{2} \xi^{\mathrm{T}} \xi+\frac{1}{2} \operatorname{tr}\left(\tilde{W}_{D}^{\mathrm{T}} \Theta_{D}^{-1} \tilde{W}_{D}\right) \\
& +\frac{1}{2} \operatorname{tr}\left(\tilde{W}_{C}^{\mathrm{T}} \Theta_{C}^{-1} \tilde{W}_{C}\right)+\frac{1}{2} \operatorname{tr}\left(\tilde{W}_{G}^{\mathrm{T}} \Theta_{G}^{-1} \tilde{W}_{G}\right)
\end{aligned}
$$

Its derivative form is

$$
\begin{aligned}
\dot{V} & =e_{q}^{\mathrm{T}} \dot{e}_{q}+e_{v}^{\mathrm{T}} D_{q} \dot{e}_{v}+\frac{1}{2} e_{v}^{\mathrm{T}} \dot{D}_{q} e_{v}+\xi^{\mathrm{T}} \dot{\xi} \\
& +\operatorname{tr}\left(\tilde{W}_{D}^{\mathrm{T}} \Theta_{D}^{-1} \dot{\tilde{W}}_{D}\right)+\operatorname{tr}\left(\tilde{W}_{C}^{\mathrm{T}} \Theta_{C}^{-1} \dot{\tilde{W}}_{C}\right)+\operatorname{tr}\left(\tilde{W}_{G}^{\mathrm{T}} \Theta_{G}^{-1} \dot{\tilde{W}}_{G}\right)
\end{aligned}
$$

Substituting (34) into (37), we have

$$
\begin{aligned}
\dot{V}= & e_{q}^{\mathrm{T}} \dot{e}_{q}-e_{v}^{\mathrm{T}}\left(-e_{q}+C_{q} e_{v}+K_{v}\left(e_{v}+\xi\right)+K_{s} \operatorname{sgn}\left(e_{v}\right)+\Delta \tau\right. \\
& +\Delta \tau_{e}+\tilde{W}_{D}^{\mathrm{T}} S_{D} \dot{\alpha}+\tilde{W}_{C}^{\mathrm{T}} S_{C} \alpha+\tilde{W}_{G}^{\mathrm{T}} S_{G}+\frac{1}{2} e_{v}^{\mathrm{T}} \dot{D}_{q} e_{v}+\xi^{\mathrm{T}} \dot{\xi} \\
& +\operatorname{tr}\left(\tilde{W}_{D}^{\mathrm{T}} \Theta_{D}^{-1} \dot{\tilde{W}}_{D}\right)+\operatorname{tr}\left(\tilde{W}_{C}^{\mathrm{T}} \Theta_{C}^{-1} \dot{\tilde{W}}_{C}\right)+\operatorname{tr}\left(\tilde{W}_{G}^{\mathrm{T}} \Theta_{G}^{-1} \dot{\tilde{W}}_{G}\right)
\end{aligned}
$$

Substituting NN updating law (32) into (38), we can obtain

$$
\begin{aligned}
\dot{V}= & e_{q}^{\mathrm{T}} \dot{e}_{q}-e_{v}^{\mathrm{T}}\left(-e_{q}+C_{q} e_{v}+K_{v}\left(e_{v}+\xi\right)+K_{s} \operatorname{sgn}\left(e_{v}\right)+\Delta \tau\right. \\
& +\Delta \tau_{e}+\tilde{W}_{D}^{\mathrm{T}} S_{D} \dot{\alpha}+\tilde{W}_{C}^{\mathrm{T}} S_{C} \alpha+\tilde{W}_{G}^{\mathrm{T}} S_{G}+\frac{1}{2} e_{v}^{\mathrm{T}} \dot{D}_{q} e_{v}+\xi^{\mathrm{T}} \dot{\xi} \\
& +\operatorname{tr}\left(\tilde{W}_{D}^{\mathrm{T}} S_{D} \dot{\alpha} e_{v}-\delta_{D} \tilde{W}_{D}^{\mathrm{T}} \tilde{W}_{D}\right)+\operatorname{tr}\left(\tilde{W}_{C}^{\mathrm{T}} \alpha e_{v}-\delta_{C} \tilde{W}_{C}^{\mathrm{T}} \tilde{W}_{C}\right) \\
& +\operatorname{tr}\left(\tilde{W}_{G} e_{v}-\delta_{G} \tilde{W}_{G}^{\mathrm{T}} \tilde{W}_{G}\right)
\end{aligned}
$$

Then, we have

$$
\begin{aligned}
\dot{V}= & e_{q}^{\mathrm{T}} \dot{e}_{q}-e_{v}^{\mathrm{T}}\left(-e_{q}+C_{q} e_{v}+K_{v}\left(e_{v}+\xi\right)+K_{s} \operatorname{sgn}\left(e_{v}\right)+\Delta \tau\right. \\
& \left.+\Delta \tau_{e}\right)+\xi^{\mathrm{T}} \dot{\xi}+\frac{1}{2} e_{v}^{\mathrm{T}} \dot{D}_{q} e_{v}-\operatorname{tr}\left(\delta_{D} \tilde{W}_{D}^{\mathrm{T}} \tilde{W}_{D}\right) \\
& -\operatorname{tr}\left(\delta_{C} \tilde{W}_{C}^{\mathrm{T}} \tilde{W}_{C}\right)-\operatorname{tr}\left(\delta_{G} \tilde{W}_{G}^{\mathrm{T}} \tilde{W}_{G}\right)
\end{aligned}
$$

Consider the following inequality [47]

$$
-\tilde{W}^{\mathrm{T}} \hat{W} \leq-\frac{1}{2} \tilde{W}^{\mathrm{T}} \tilde{W}+\frac{1}{2} W^{* \mathrm{~T}} W^{*}
$$

Since

$$
\begin{aligned}
\xi^{\mathrm{T}} \Delta \tau & \leq \frac{1}{2} \xi^{\mathrm{T}} \xi+\frac{1}{2} \Delta \tau^{\mathrm{T}} \Delta \tau \\
-e_{v}^{\mathrm{T}} \Delta \tau_{e} & \leq \frac{1}{2} e_{v}^{\mathrm{T}} e_{v}+\frac{1}{2} \Delta \tau_{e}^{\mathrm{T}} \Delta \tau_{e}
\end{aligned}
$$

From (30), we have

$$
\begin{aligned}
\xi^{\mathrm{T}} \dot{\xi} & \leq-\xi^{\mathrm{T}} K_{\xi} \xi-\left|e_{v}^{\mathrm{T}} \Delta \tau\right|-\frac{1}{2} \Delta \tau^{\mathrm{T}} \Delta \tau+\xi^{\mathrm{T}} \Delta \tau \\
& \leq-\xi^{\mathrm{T}} K_{\xi} \xi-\left|e_{v}^{\mathrm{T}} \Delta \tau\right|+\frac{1}{2} \xi^{\mathrm{T}} \xi
\end{aligned}
$$

Then, combing the (43) and the property 2 , the derivative form (40) can be

$$
\begin{aligned}
\dot{V} \leq & e_{q}^{\mathrm{T}} \dot{e}_{q}-e_{v}^{\mathrm{T}}\left(-e_{q}+K_{v}\left(e_{v}+\xi\right)+K_{s} \operatorname{sgn}\left(e_{v}\right)\right. \\
& \left.+\Delta \tau+\Delta \tau_{e}\right)-\xi^{\mathrm{T}} K_{\xi} \xi-\left|e_{v}^{\mathrm{T}} \Delta \tau\right|+\frac{1}{2} \xi^{\mathrm{T}} \xi \\
& -\operatorname{tr}\left(\delta_{D} \tilde{W}_{D}^{\mathrm{T}} \tilde{W}_{D}\right)-\operatorname{tr}\left(\delta_{C} \tilde{W}_{C}^{\mathrm{T}} \tilde{W}_{C}\right)-\operatorname{tr}\left(\delta_{G} \tilde{W}_{G}^{\mathrm{T}} \tilde{W}_{G}\right)
\end{aligned}
$$



(44)

Considering the error signal $e_{v}=\dot{e}_{q}+K e_{q}$, We can simplify

$$
\begin{aligned}
\dot{V} \leq & e_{q}^{\mathrm{T}} \dot{e}_{q}-e_{v}^{\mathrm{T}}\left(-e_{q}+K_{v}\left(e_{v}+\xi\right)\right) \\
& -e_{v}^{\mathrm{T}} \Delta \tau_{e}-\xi^{\mathrm{T}} K_{\xi} \xi-\left|e_{v}^{\mathrm{T}} \Delta \tau\right|+\frac{1}{2} \xi^{\mathrm{T}} \xi \\
& -\operatorname{tr}\left(\delta_{D} \tilde{W}_{D}^{\mathrm{T}} \tilde{W}_{D}\right)-\operatorname{tr}\left(\delta_{C} \tilde{W}_{C}^{\mathrm{T}} \tilde{W}_{C}\right)-\operatorname{tr}\left(\delta_{G} \tilde{W}_{G}^{\mathrm{T}} \tilde{W}_{G}\right)
\end{aligned}
$$

Unfolding (45), we have

$$
\begin{aligned}
\dot{V} \leq & e_{q}^{\mathrm{T}} \dot{e}_{v}-e_{q}^{\mathrm{T}} K \dot{e}_{q}+e_{v}^{\mathrm{T}} e_{q}-e_{v}^{\mathrm{T}} K_{v} e_{v} \\
& -e_{v}^{\mathrm{T}} K_{v} \xi+\frac{1}{2} e_{v}^{\mathrm{T}} e_{v}+\frac{1}{2} \Delta \tau_{e}^{\mathrm{T}} \Delta \tau_{e}-\xi^{\mathrm{T}} K_{\xi} \xi+\frac{1}{2} \xi^{\mathrm{T}} \xi \\
& -\operatorname{tr}\left(\delta_{D} \tilde{W}_{D}^{\mathrm{T}} \tilde{W}_{D}\right)-\operatorname{tr}\left(\delta_{C} \tilde{W}_{C}^{\mathrm{T}} \tilde{W}_{C}\right)-\operatorname{tr}\left(\delta_{G} \tilde{W}_{G}^{\mathrm{T}} \tilde{W}_{G}\right) \\
\leq & -e_{q}^{\mathrm{T}} K e_{q}-\frac{1}{2} \xi^{\mathrm{T}}\left(2 K_{\xi}-I-K_{v}^{\mathrm{T}} k_{v}\right) \xi-e_{v}^{\mathrm{T}}\left(K_{v}-I\right) e_{v} \\
& -\operatorname{tr}\left(\delta_{D} \tilde{W}_{D}^{\mathrm{T}} \tilde{W}_{D}\right)-\operatorname{tr}\left(\delta_{C} \tilde{W}_{C}^{\mathrm{T}} \tilde{W}_{C}\right)-\operatorname{tr}\left(\delta_{G} \tilde{W}_{G}^{\mathrm{T}} \tilde{W}_{G}\right)+C
\end{aligned}
$$

where $C=\frac{1}{2} \Delta \tau_{e}^{\mathrm{T}} \Delta \tau_{e}$. To ensure the stability of the closedloop system, the parameters should satisfy : $K_{v}-I \geq 0$ and $2 K_{\xi}-I-K_{v}^{\mathrm{T}} K_{v} \geq 0$.

Let us define the variable $\varsigma$ comprised of $e_{q}, \xi, e_{v}, \tilde{W}_{D}, \tilde{W}_{C}, \tilde{W}_{G}$ and the derivative form of $V$ can be rewritten as $\dot{V}(\varsigma) \leq-A \varphi(\varsigma)+C$, where $A$ and $C$ are positive constants. There exists a invariant set $L(\varsigma)$, that makes: $-A \varphi(\varsigma)+C<0$ when $\varsigma$ starts outside of the set $L(\varsigma)$. At this time, since $\dot{V}(\varsigma)<0, V(\varsigma)$ decreases so that $\varsigma$ will enter into $L(\varsigma)$ within a period of time and remain afterwards. Therefore, the state variable $\varsigma$ satisfies uniformly ultimately bounded(UUB) stablity and approaches to a bounded compact set near zero.

The invariant set $L(\varsigma)$ can be defined as follows

$$
\begin{aligned}
\Omega_{s}= & \left\{\left(\left\|\tilde{W}_{D}\right\|,\left\|\tilde{W}_{C}\right\|,\left\|\tilde{W}_{G}\right\|,\left\|e_{q}\right\|,\left\|e_{v}\right\|, \xi\right), \mid\right. \\
& \frac{e_{q}^{\mathrm{T}} K e_{q}}{C}+\frac{\frac{1}{2} \xi^{\mathrm{T}}\left(2 K_{\xi}-I-K_{v}^{\mathrm{T}} k_{v}\right) \xi}{C}+\frac{e_{v}^{\mathrm{T}}\left(K_{v}-I\right) e_{v}}{C} \\
& \left.\frac{\sigma_{D}\left\|\tilde{W}_{D}\right\|^{2}}{C}+\frac{\sigma_{C}\left\|\tilde{W}_{C}\right\|^{2}}{C}+\frac{\sigma_{G}\left\|\tilde{W}_{G}\right\|^{2}}{C} \leq 1\right\}
\end{aligned}
$$

\section{REFERENCES}

[1] K. OHNISHI, "H observed based force control without force sensor," IEEE Proc. IECON'91, pp. 1049-1054, 1991.

[2] P. Hacksel and S. Salcudean, "Estimation of environment forces and rigid-body velocities using observers," in Robotics and Automation, 1994. Proceedings., 1994 IEEE International Conference on. IEEE, 1994, pp. 931-936.

[3] A. Alcocer, A. Robertsson, A. Valera, and R. Johansson, "Force estimation and control in robot manipulators," IFAC Proceedings Volumes, vol. 36, no. 17, pp. 55-60, 2003.

[4] K. S. Eom, I. H. Suh, W. K. Chung, and S.-R. Oh, "Disturbance observer based force control of robot manipulator without force sensor," in Robotics and Automation, 1998. Proceedings. 1998 IEEE International Conference on, vol. 4. IEEE, 1998, pp. 3012-3017.

[5] M. V. Damme, P. Beyl, B. Vanderborght, V. Grosu, R. V. Ham, I. Vanderniepen, A. Matthys, and D. Lefeber, "Estimating robot endeffector force from noisy actuator torque measurements," in 2011 IEEE International Conference on Robotics and Automation, May 2011, pp. $1108-1113$.

[6] A. Wahrburg, E. Morara, G. Cesari, B. Matthias, and H. Ding, "Cartesian contact force estimation for robotic manipulators using kalman filters and the generalized momentum," in 2015 IEEE International Conference on Automation Science and Engineering (CASE), Aug 2015, pp. 12301235 .
[7] J. J. Craig and M. H. Raibert, "A systematic method of hybrid position/force control of a manipulator," in Computer Software and Applications Conference, 1979. Proceedings. COMPSAC 79. The IEEE Computer Society's Third International. IEEE, 1979, pp. 446-451.

[8] J. E. Colgate and N. Hogan, "Robust control of dynamically interacting systems," International journal of Control, vol. 48, no. 1, pp. 65-88, 1988.

[9] R. Colbaugh, H. Seraji, and K. Glass, "Direct adaptive impedance control of robot manipulators," Journal of Robotic Systems, vol. 10, no. 2, pp. 217-248, 1993.

[10] S. P. Buerger and N. Hogan, "Complementary stability and loop shaping for improved human-robot interaction," IEEE Transactions on Robotics, vol. 23, no. 2, pp. 232-244, 2007.

[11] F. Caccavale, P. Chiacchio, A. Marino, and L. Villani, "Six-dof impedance control of dual-arm cooperative manipulators," IEEE/ASME Transactions on Mechatronics, vol. 13, no. 5, pp. 576-586, Oct 2008.

[12] M. T. Mason, "Compliance and force control for computer controlled manipulators," IEEE Transactions on Systems, Man, and Cybernetics, vol. 11, no. 6, pp. 418-432, June 1981.

[13] H. Seraji, "Adaptive admittance control: an approach to explicit force control in compliant motion," in Proceedings of the 1994 IEEE International Conference on Robotics and Automation, May 1994, pp. 27052712 vol.4.

[14] T. Zhang, C. L. P. Chen, L. Chen, X. Xu, and B. Hu, "Design of highly nonlinear substitution boxes based on i-ching operators," IEEE Transactions on Cybernetics, pp. 1-10, 2018.

[15] Y. Wang, H. R. Karimi, H. Shen, Z. Fang, and M. Liu, "Fuzzy-modelbased sliding mode control of nonlinear descriptor systems," IEEE Transactions on Cybernetics, pp. 1-11, 2018.

[16] Y. Wang, H. R. Karimi, H. Lam, and H. Shen, "An improved result on exponential stabilization of sampled-data fuzzy systems," IEEE Transactions on Fuzzy Systems, vol. 26, no. 6, pp. 3875-3883, Dec 2018.

[17] C. Yang, Y. Jiang, Z. Li, W. He, and C. Su, "Neural control of bimanual robots with guaranteed global stability and motion precision," IEEE Transactions on Industrial Informatics, vol. 13, no. 3, pp. 1162-1171, June 2017.

[18] C. Yang, X. Wang, L. Cheng, and H. Ma, "Neural-learning-based telerobot control with guaranteed performance," IEEE Transactions on Cybernetics, vol. 47, no. 10, pp. 3148-3159, Oct 2017.

[19] Z. Li, J. Li, S. Zhao, Y. Yuan, Y. Kang, and C. L. P. Chen, "Adaptive neural control of a kinematically redundant exoskeleton robot using brain-machine interfaces," IEEE Transactions on Neural Networks and Learning Systems, pp. 1-14, 2018.

[20] L. Zhang, Z. Li, and C. Yang, "Adaptive neural network based variable stiffness control of uncertain robotic systems using disturbance observer," IEEE Transactions on Industrial Electronics, vol. 64, no. 3, pp. 2236-2245, March 2017.

[21] S. Zhang, Y. Dong, Y. Ouyang, Z. Yin, and K. Peng, "Adaptive neural control for robotic manipulators with output constraints and uncertainties," IEEE Transactions on Neural Networks and Learning Systems, vol. 29, no. 11, pp. 5554-5564, Nov 2018.

[22] G. Li, J. Na, D. P. Stoten, and X. Ren, "Adaptive neural network feedforward control for dynamically substructured systems," IEEE Transactions on Control Systems Technology, vol. 22, no. 3, pp. 944-954, May 2014.

[23] H. Liu, Y. Pan, S. Li, and Y. Chen, "Adaptive fuzzy backstepping control of fractional-order nonlinear systems," IEEE Transactions on Systems, Man, and Cybernetics: Systems, vol. 47, no. 8, pp. 2209-2217, Aug 2017.

[24] C. L. P. Chen, T. Zhang, L. Chen, and S. C. Tam, "I-ching divination evolutionary algorithm and its convergence analysis," IEEE Transactions on Cybernetics, vol. 47, no. 1, pp. 2-13, Jan 2017.

[25] C. Juang, M. Lai, and W. Zeng, "Evolutionary fuzzy control and navigation for two wheeled robots cooperatively carrying an object in unknown environments," IEEE Transactions on Cybernetics, vol. 45, no. 9, pp. 1731-1743, Sept 2015.

[26] N. O. Perez-Arancibia, T. C. Tsao, and J. S. Gibson, "Saturation-induced instability and its avoidance in adaptive control of hard disk drives," IEEE Transactions on Control Systems Technology, vol. 18, no. 2, pp. 368-382, March 2010.

[27] G. Tao and P. V. Kokotovic, Adaptive control of systems with actuator and sensor nonlinearities. John Wiley \& Sons, Inc., 1996.

[28] S. S. Ge, C. C. Hang, T. H. Lee, and T. Zhang, Stable adaptive neural network control. Springer Science \& Business Media, 2013, vol. 13.

[29] W. Gao and R. R. Selmic, "Adaptive neural network output feedback control of nonlinear systems with actuator saturation," in Proceedings of the 44th IEEE Conference on Decision and Control, Dec 2005, pp. 5522-5527.

[30] C. Wen, J. Zhou, Z. Liu, and H. Su, "Robust adaptive control of uncertain nonlinear systems in the presence of input saturation and external disturbance," IEEE Transactions on Automatic Control, vol. 56, no. 7, pp. 1672-1678, July 2011. 
[31] W. He, Y. Dong, and C. Sun, "Adaptive neural impedance control of a robotic manipulator with input saturation," IEEE Transactions on Systems, Man, and Cybernetics: Systems, vol. 46, no. 3, pp. 334-344, 2016.

[32] G. Peng, C. Yang, W. He, Z. Li, and D. Kuang, "Neural-learning enhanced admittance control of a robot manipulator with input saturation," in 2017 Chinese Automation Congress (CAC), Oct 2017, pp. 104-109.

[33] C. Wang, Y. Li, S. S. Ge, K. P. Tee, and T. H. Lee "Continuous critic learning for robot control in physical human-robot interaction," in 2013 13th International Conference on Control, Automation and Systems (ICCAS 2013), Oct 2013, pp. 833-838.

[34] B. Siciliano and O. Khatib, Springer handbook of robotics. Springer, 2016.

[35] S. S. Ge, C. C. Hang, and L. C. Woon, "Adaptive neural network control of robot manipulators in task space," IEEE Transactions on Industrial Electronics, vol. 44, no. 6, pp. 746-752, Dec 1997.

[36] Z. Chen, Z. Li, and C. L. P. Chen, "Adaptive neural control of uncertain mimo nonlinear systems with state and input constraints," IEEE Transactions on Neural Networks and Learning Systems, vol. 28 , no. 6, pp. 1318-1330, June 2017.

[37] A. Colom, D. Pardo, G. Aleny, and C. Torras, "External force estimation during compliant robot manipulation," in 2013 IEEE International Conference on Robotics and Automation, May 2013, pp. 3535-3540.

[38] A. Smith, C. Yang, C. Li, H. Ma, and L. Zhao, "Development of a dynamics model for the baxter robot," in 2016 IEEE International Conference on Mechatronics and Automation, Aug 2016, pp. 1244 1249.

[39] F. A. Shaik and S. Purwar, "A nonlinear state observer design for 2dof twin rotor system using neural networks," in 2009 International Conference on Advances in Computing, Control, and Telecommunication Technologies, Dec 2009, pp. 15-19.

[40] A. C. Smith, F. Mobasser, and K. Hashtrudi-Zaad, "Neural-networkbased contact force observers for haptic applications," IEEE Transactions on Robotics, vol. 22, no. 6, pp. 1163-1175, Dec 2006.

[41] W. He and S. S. Ge, "Cooperative control of a nonuniform gantry crane with constrained tension," Automatica, vol. 66, pp. 146-154, 2016.

[42] P. Korba, R. Babuska, H. B. Verbruggen, and P. M. Frank, "Fuzzy gain scheduling: controller and observer design based on lyapunov method and convex optimization," IEEE transactions on fuzzy systems, vol. 11, no. 3, pp. 285-298, 2003.

[43] K. S. Narendra and J. Balakrishnan "A common lyapunov function for stable lti systems with commuting a-matrices," IEEE Transactions on Automatic Control, vol. 39, no. 12, pp. 2469-2471, Dec 1994.

[44] P. Korba, "A gain-scheduling approach to model-based fuzzy control," at-Automatisierungstechnik Methoden und Anwendungen der Steuerungs-, Regelungs-und Informationstechnik, vol. 49, no. 5/2001, p. 245_1, 2001.

[45] P. Korba and P. Frank, "An applied optimization-based gain-scheduled fuzzy control," in American Control Conference, 2000. Proceedings of the 2000, vol. 5. IEEE, 2000, pp. 3383-3387.

[46] I. Ranatunga, F. L. Lewis, D. O. Popa, and S. M. Tousif, "Adaptive admittance control for human-robot interaction using model reference design and adaptive inverse filtering." IEEE Trans. Contr. Sys. Techn., vol. 25 , no. 1 , pp. $278-285,2017$

[47] W. H. Young, "On classes of summable functions and their fourier series," Proceedings of the Royal Society of London. Series A, Containing Papers of a Mathematical and Physical Character, vol. 87, no. 594, pp. 225-229, 1912.

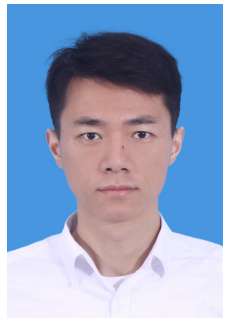

Guangzhu Peng received the B.Eng. degree from Yangtze University, Jingzhou, China, in 2014, the M.Eng. degree in Automation Science and Engineering from South China University of Technology, Guangzhou, China, in 2018. He is currently pursuing the Ph.D. degree in computer science with the Faculty of Science and Technology, University of Macau, Macau, China.

His current research interests include robotics, human-robot interaction, intelligent control, etc.

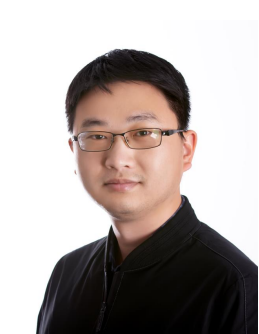

Chenguang Yang (M'10-SM'16) is a Professor of Robotics. He received the Ph.D. degree in control engineering from the National University of Singapore, Singapore, in 2010 and performed postdoctoral research in human robotics at Imperial College London, London, UK from 2009 to 2010 He has been awarded EU Marie Curie International Incoming Fellowship, UK EPSRC UKRI Innovation Fellowship, and the Best Paper Award of the IEEE Transactions on Robotics as well as over ten conference Best Paper Awards. His research interest lies in human robot interaction and intelligent system design.

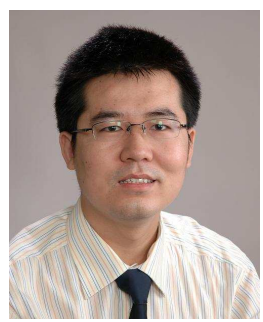

Wei He (S'09-M'12-SM'16) received his B.Eng. and his M.Eng. degrees from College of Automation Science and Engineering, South China University of Technology (SCUT), China, in 2006 and 2008, respectively, and his $\mathrm{PhD}$ degree from Department of Electrical \& Computer Engineering, the National University of Singapore (NUS), Singapore, in 2011. He is currently working as a full professor in School of Automation and Electrical Engineering, University of Science and Technology Beijing, Beijing, China. He has co-authored 3 books published in Springer and published over 100 international journal and conference papers. He was awarded a Newton Advanced Fellowship from the Royal Society, UK in 2017. He was a recipient of the IEEE SMC Society Andrew P. Sage Best Transactions Paper Award in 2017. He is serving the Chair of IEEE SMC Society Beijing Capital Region Chapter. He is serving as an Associate Editor of IEEE Transactions on Neural Networks and Learning Systems, IEEE Transactions on Control Systems Technology, IEEE Transactions on Systems, Man, and Cybernetics: Systems, Assembly Automation, IEEE/CAA Journal of Automatica Sinica, Neurocomputing, and an Editor of Journal of Intelligent \& Robotic Systems. His current research interests include robotics, distributed parameter systems and intelligent control systems.

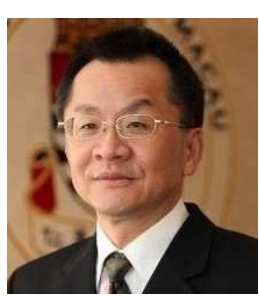

C. L. Philip Chen (S'88-M'88-SM'94-F'07) is a chair professor of the Department of Computer and Information Science, Faculty of Science and Technology, University of Macau, Macau, China. Being a Program Evaluator of the Accreditation Board of Engineering and Technology Education (ABET) in the U.S. for computer engineering, electrical engineering, and software engineering programs, he successfully architects the University of Macau's Engineering and Computer Science programs receiving accreditations from Washington/Seoul Accord through Hong Kong Institute of Engineers (HKIE), of which is considered as his utmost contribution in engineering/computer science education for Macau as the former Dean of the Faculty.

His current research interests include systems, cybernetics, and computational intelligence. Dr. Chen was the IEEE SMC Society President from 2012 to 2013 and is now a Vice President of Chinese Association of Automation (CAA). He is a Fellow of IEEE, AAAS, IAPR, CAA, and HKIE. He is the editor-in-chief of the IEEE Transaction on Systems, Man, and Cybernetics: Systems, and an associate editor of IEEE Transactions on Fuzzy Systems and IEEE Transactions on Cybernetics. He was the Chair of TC 9.1 Economic and Business Systems of International Federation of Automatic Control (IFAC), 2015-2017. He received 2016 Outstanding Electrical and Computer Engineers award from his alma mater, Purdue University, after he graduated from the University of Michigan, Ann Arbor, Michigan, USA. 